\title{
Comments on the Article "Arthroscopic Treatment for Femoroacetabular Impingement with Extraspinal Diffuse Idiopathic Skeletal Hyperostosis": To the Editor
}

\author{
Jiangen Lian, MD, Junfeng Lian, BS, Mingjin Zhong, MD* \\ Department of Orthopaedic Surgery, Dabu People's Hospital, Dabu, \\ * Department of Sports Medicine, The First Affiliated Hospital of Shenzhen University, Health Science Center; \\ Shenzhen Second People's Hospital, Shenzhen, China
}

\section{To the Editor:}

With the rapid growth of the use of hip arthroscopy and strong evidence on its clinical efficacy and safety in the treatment of femoroacetabular impingement (FAI), we read with great interest the recent article entitled "Arthroscopic treatment for femoroacetabular impingement with extraspinal diffuse idiopathic skeletal hyperostosis" by Hwang et al. ${ }^{1)}$

To the best of our knowledge, it is the first paper that studied the clinical outcomes of hip arthroscopy in patients with extraspinal diffuse idiopathic skeletal hyperostosis (DISH) that involves the hip joint. This retrospective study suggests that extraspinal DISH involving the hip joint could lead to FAI and arthroscopic treatment could provide good outcomes. The study also emphasizes that FAI patients who have painful hip and limitation of range of motion should also be evaluated to determine whether they have extraspinal DISH involving the hip. ${ }^{1)}$ We really appreciate the excellent work of the authors. However, there are some issues worth to be explored.

Ossification with bony overgrowth in extraspinal DISH mostly involves the acetabular rim of the hip joint, which leads to pincer-type FAI. Pincer FAI occurs in two forms: focal and global. ${ }^{2)}$ Focal acetabular overcoverage can exist in the anterosuperior acetabulum (as shown in Fig. 2) ${ }^{1)}$ with a center-edge angle (CEA; Wiberg angle) of $25^{\circ}-39^{\circ}$. Global FAI has more generalized and typically more extreme acetabular overcoverage, resulting in a CEA of $40^{\circ}$ or greater with protrusio acetabuli or coxa profun$\mathrm{da}^{2)}$ (as shown in Fig. 1). ${ }^{1)}$ The reduction in CEA is a more important determinant of hip function than is the magnitude of the preoperative or postoperative CEA.) Therefore, we think the preoperative and postoperative CEAs should have been carefully evaluated in the study.
The authors described that the central compartment (CC) of the hip joint was addressed first during the hip arthroscopic surgery. However, because of severe acetabular overcoverage, joint space narrowing, and early stage osteoarthritis (Fig. 1), ${ }^{1)}$ traction is usually very difficult to achieve, and the entry needle access into the CC is often blocked from the anterolateral or anterior portal. ${ }^{4)}$ In this condition, the peripheral compartment (PC) of the hip joint would be explored first as Dienst et al. ${ }^{5}$ described: the patient is placed on a standard traction table without joint traction and with the hip and knee semiflexed. The entry needle is used for confirmation of entry to the joint by lateral and caudal displacement of the femoral head under fluoroscopy. The PC is easily explored by portal exchange and internal and external rotation of the hip. Then, acetabuloplasty and femoroplasty from PC are performed until sufficient to permit CC access. After PC manipulation, the hip and knee are extended, the CC is addressed with traction.

We are convinced that hip arthroscopy for the treatment of patients with extraspinal DISH involving the hip joint will show good clinical results without any intraor postoperative complications related to the surgical procedure. However, extraspinal DISH is a progressive disease characterized by abnormal calcification and ossification around joints, which may be one of the causes of secondary osteoarthritis. In patients with FAI, older age (>45 years), elevated BMI (>24.5 kg/m $\left.{ }^{2}\right)$, osteoarthritic changes, and increased CEA are predictors of negative outcomes after hip arthroscopy. ${ }^{6}$ Obviously, the 17 hips (12 patients) met one or more of those conditions. Therefore, the degree of hip arthritis and rates of revision surgery and conversion to total hip arthroplasty should be assessed in the long term. We look forward to seeing the long-term 
Letter to the Editor

Clinics in Orthopedic Surgery • Vol. 12, No. 2, $2020 \bullet$ www.ecios.org

follow-up outcomes of the study in terms of patients' function, painful symptoms, and complications. We respect the great contribution of the authors and are much interested in the authors' thoughts on these issues.

\section{CONFLICT OF INTEREST}

No potential conflict of interest relevant to this article was reported.
Received September 10, 2019; Accepted September 17, 2019

Correspondence to: Mingjin Zhong, MD

Department of Sports Medicine, The First Affiliated Hospital of Shenzhen University, Health Science Center; Shenzhen Second People's Hospital, 3002 Sungang West Road, Futian District, Shenzhen 518000, China Tel: +86-0755-83366388, Fax: +86-0755-83356952

E-mail: sportsmedzhong@sina.com

https://doi.org/10.4055/cios19138

\section{REFERENCES}

1. Hwang JM, Hwang DS, Kang C, et al. Arthroscopic treatment for femoroacetabular impingement with extraspinal diffuse idiopathic skeletal hyperostosis. Clin Orthop Surg. 2019;11(3):275-81.

2. Matsuda DK, Gupta N, Burchette RJ, Sehgal B. Arthroscopic surgery for global versus focal pincer femoroacetabular impingement: are the outcomes different? J Hip Preserv Surg. 2015;2(1):42-50.

3. Sanders TL, Reardon P, Levy BA, Krych AJ. Arthroscopic treatment of global pincer-type femoroacetabular impingement. Knee Surg Sports Traumtol Arthrosc. 2017;25(1):31-5.
4. Matsuda DK, Gupta N, Hanami D. Hip arthroscopy for challenging deformities: global pincer femoroacetabular impingement. Arthrosc Tech. 2014;3(2):e197-204.

5. Dienst M, Godde S, Seil R, Hammer D, Kohn D. Hip arthroscopy without traction: in vivo anatomy of the peripheral hip joint cavity. Arthroscopy. 2001;17(9):924-31.

6. Sogbein OA, Shah A, Kay J, et al. Predictors of outcomes after hip arthroscopic surgery for femoroacetabular impingement: a systematic review. Orthop J Sports Med. 2019;7(6):2325967119848982. 
Letter to the Editor

Clinics in Orthopedic Surgery • Vol. 12, No. 2, $2020 \bullet$ www.ecios.org

Jung-Mo Hwang, Deuk-Soo Hwang, Chan Kang, Woo-Yong Lee, Gi-Soo Lee, Jeong-Kil Lee, Yun-Ki Kim, Reply:

We thank the readers for their interest in our manuscript published in September 2019 issue of Clinics in Orthopedic Surgery. ${ }^{1)}$ We would like to answer the points raised by the authors of the letter to editor as follows:

First, we agree that the preoperative and postoperative lateral center-edge angles (CEAs) of Wiberg need to be carefully evaluated. However, we did not evaluate the preoperative and postoperative CEAs for some reasons. In the paper you cited, Sanders et al. ${ }^{2)}$ reported that neither preoperative nor postoperative CEA was consistently associated with lower hip function. And many other previous papers reported excellent hip function after arthroscopic treatment of patients with global overcoverage, even when postoperative CEA was greater than $40^{\circ}{ }^{3,4)}$ Sanders et al. ${ }^{2)}$ reported that a reduction in CEA is a more important determinant of hip function than the magnitude of the preoperative or postoperative CEA. If extraspinal diffuse idiopathic skeletal hyperostosis (DISH) involves the hip joint, particularly the superior acetabulum, hip pain and range of motion (ROM) limitation (femoroacetabular impingement symptoms) could be present. ${ }^{5,6)}$ We believe that assessing the magnitude of the preoperative and/or postoperative CEA is useful for evaluating radiologic results after arthroscopic decompression of hyperostosis of the acetabulum; however, it does not seem to reflect hip function after surgery. In our experience, many patients expect to have improved ROM of the hip joint after surgery. For these reasons, we evaluated the change in hip joint ROM, not the magnitude of the preoperative and postoperative CEA.

Second, we completely agree that the peripheral compartment (PC) of the hip joint would be explored first when the entry needle access into the central compartment (CC) is blocked because of severe acetabular overcoverage and joint space narrowing. We also experienced this several times in case of Legg-Calvé-Perthes deformity, the peripheral type of ankylosing spondylitis, etc. In these conditions, we also accessed the PC of the hip joint first. Fortunately, in our study, the CC could be approached first because of proper widening of the joint space by traction. Once again thank you for the good surgical technique advice.

Finally, extraspinal DISH seems to be a slowly progressing disease characterized by abnormal calcification and/or ossification of soft tissues around joints, which may be one of the causes of hyperostosis and atypical osteoarthritis. ${ }^{7-9)}$ In our paper, the clinical outcomes were good at a minimum of 2 years postoperatively. However, long-term results need to be assessed to ascertain the effectiveness of the treatment.

\section{CONFLICT OF INTEREST}

No potential conflict of interest relevant to this article was reported.

Correspondence to: Deuk-Soo Hwang, MD

Department of Orthopaedic Surgery, Chungnam National University School of Medicine, 266 Munhwa-ro, Jung-gu, Daejeon 35015, Korea

Tel: +82-42-338-2480, Fax: +82-42-338-2482

E-mail: dshwang@cnu.ac.kr

https://doi.org/10.4055/cios19138re

\section{REFERENCES}

1. Hwang JM, Hwang DS, Kang C, et al. Arthroscopic treatment for femoroacetabular impingement with extraspinal diffuse idiopathic skeletal hyperostosis. Clin Orthop Surg. 2019;11(3):275-81.

2. Sanders TL, Reardon P, Levy BA, Krych AJ. Arthroscopic treatment of global pincer-type femoroacetabular impingement. Knee Surg Sports Traumatol Arthrosc. 2017;25(1):315.

3. Matsuda DK. Protrusio acetabuli: contraindication or indication for hip arthroscopy? And the case for arthroscopic treatment of global pincer impingement. Arthroscopy. 2012;28(6):882-8.
4. Safran MR, Epstein NP. Arthroscopic management of protrusio acetabuli. Arthroscopy. 2013;29(11):1777-82.

5. Mader R, Sarzi-Puttini P, Atzeni F, et al. Extraspinal manifestations of diffuse idiopathic skeletal hyperostosis. Rheumatology (Oxford). 2009;48(12):1478-81.

6. Terzi R. Extraskeletal symptoms and comorbidities of diffuse idiopathic skeletal hyperostosis. World J Clin Cases. 2014;2(9):422-5.

7. Haller J, Resnick D, Miller CW, et al. Diffuse idiopathic skeletal hyperostosis: diagnostic significance of radiographic abnormalities of the pelvis. Radiology. 1989;172(3):835-9. 
Letter to the Editor

Clinics in Orthopedic Surgery • Vol. 12, No. 2, $2020 \bullet$ www.ecios.org

8. Resnick D, Shaul SR, Robins JM. Diffuse idiopathic skeletal hyperostosis (DISH): Forestier's disease with extraspinal manifestations. Radiology. 1975;115(3):513-24.
9. Utsinger PD. Diffuse idiopathic skeletal hyperostosis. Clin Rheum Dis. 1985;11(2):325-51. 